(1) Check for undates

СОВРЕМЕННЫЕ ВОЗМОЖНОСТИ ПРИМЕНЕНИЯ СТЕРОИДНОГО ПРОФИЛЯ МОЧИ ДЛЯ ДИАГНОСТИКИ АДРЕНОКОРТИКАЛЬНОГО РАКА

(С) И.Е. Щербаков ${ }^{1 *}$, Р.А. Черников ${ }^{1}$, В.Ф. Русаков ${ }^{1,2}$, Е.А. Федоров ${ }^{1}$, Ю.Н. Федотов ${ }^{1}$, И.В. Саблин ${ }^{1}$, И.К. Чинчук ${ }^{1}$, И.В. Слепцов ${ }^{1}$, Л.М. Краснов ${ }^{1}$, Д.В. Реброва' ${ }^{1}$ М.А. Уварова ${ }^{1}$, А.В. Иванов ${ }^{1}$

1Санкт-Петербургский государственный университет, Клиника высоких медицинских технологий им. Н.И. Пирогова, Санкт-Петербург, Россия

${ }^{2}$ Военно-медицинская академия им. С.М. Кирова, Санкт-Петербург, Россия

ОБОСНОВАНИЕ. Важную роль в определении злокачественной природы новообразований надпочечников играют лучевые методы исследования. Чувствительность и специфичность этих методов крайне высоки. Вместе с тем, при высокой диагностической эффективности современных лучевых методов визуализации опухолей надпочечников, для постановки точного клинического диагноза также необходимо проведение гормональных исследований. Нарушения стероидного профиля мочи выявляются при различной патологии надпочечников (первичном гиперальдостеронизме, гиперкортицизме, врожденной гиперплазии коры надпочечников и адренокортикальном раке). Исследование стероидного профиля мочи у пациентов с диагностированными новообразованиями надпочечников предназначено в первую очередь для подтверждения или опровержения риска адренокортикального рака. При этом в вопросе точности и значимости исследований стероидного профиля мочи в медицинском сообществе к настоящему времени накопилось достаточное количество разногласий.

ЦЕЛЬ. Исследование направлено на определение границ точности применения стероидного профиля мочи для диагностики адренокортикального рака.

МЕТОДЫ. Всего исследовались 62 пробы у 58 пациентов с морфологически подтвержденными новообразованиями надпочечников. Исследование носило характер слепого проспективного. Для повышения точности исследования из 58 больных были отобраны 30 пациентов с адренокортикальными аденомами ( $\mathrm{n=17)}$ и адренокортикальным раком $(\mathrm{n}=13)$. Стероидный профиль мочи был изучен методом газовой хромато-масс-спектрометрии. В целях оценки информативности стероидного профиля проводилось определение чувствительности, специфичности и точности методики для диагностики адренокортикальной карциномы.

РЕЗУЛЬТАТЫ. Оценены возможности определения стероидного профиля мочи для диагностики адренокортикального рака. Чувствительность метода составила 46,2\%, специфичность и точность - 70,6\% и 60\% соответственно. Наиболее достоверными маркерами адренокортикального рака являются тетрагидро-11-дезоксикортизол и дегидроэпиандростерон (38,5\% наблюдений).

ЗАКЛЮчЕНИЕ. Настоящее исследование демонстрирует относительно невысокие показатели диагностической эффективности определения стероидного профиля мочи как метода первичной диагностики адренокортикального рака. Особенно это видно по сравнению с лучевыми методами диагностики. Интерпретация методики сложна и доступна лишь специалистам с чрезвычайно высокой квалификацией, что затрудняет распространение и широкое применение этого метода обследования в клинической практике. Вместе с тем определение стероидного профиля мочи в перспективе (после дополнительного изучения) может быть использовано как вспомогательный метод диагностики, в ряде случаев определяющей лечебную тактику у пациентов, перенесших адреналэктомию по поводу адренокортикального рака.

КЛЮЧЕВЫЕ СЛОВА: адренокортикальный рак; стероидный профиль мочи; газовая хромато-масс-спектрометрия; стероидогенез.

\title{
MODERN POSSIBILITIES OF THE URINE STEROID PROFILE TESTING APPLYING FOR THE ADRENOCORTICAL CANCER DIAGNOSIS
}

(c) Ilya E. Shcherbakov, Roman A. Chernikov, Vladimir F. Rusakov, Elysey A. Fedorov, Uriy N. Fedotov, Ilya V. Sablin, Igor K. Chinchuk, Ilya V. Slepcov, Leonid M. Krasnov, Dina V. Rebrova, Marina A. Uvarova, Andrey V. Ivanov

'Saint Petersburg State University Hospital, St. Petersburg, Russia ${ }^{2}$ Military Medical Academy named after SM Kirov, St. Petersburg, Russia

BACKGROUND: X-ray diagnostics methods are important in detection of adrenal neoplasms malignant nature. The sensitivity and specificity of these methods are high enough. However the hormonal tests are also necessary to make an accurate clinical diagnosis with the high diagnostic efficiency of modern X-ray methods for adrenal tumors diagnosing. The urine steroid profile violations are detected with the adrenal glands various pathologies (primary hyperaldosteronism, 
hypercorticism, congenital hyperplasia of the adrenal cortex and adrenocortical cancer). Urine steroid profile tests in patients with diagnosed adrenal neoplasms are intended primarily to confirm or refute the adrenocortical cancer risk. At the same time in the medical community to date there are a number of disagreements accumulated regarding the accuracy and significance of the urine steroid profile tests.

AIMS: The study aims to determine the urine steroid profile determination accuracy limits for the adrenocortical cancer diagnosis.

MATERIALS AND METHODS: In total 62 samples were tested for urine steroid profile by gas chromatography-mass spectrometry. 58 patients had morphologically confirmed adrenal neoplasms. The study was blind prospective. To increase the study accuracy the 30 patients with adrenocortical adenomas $(n=17)$ and adrenocortical cancer $(n=13)$ were selected out of 58 tested persons. The sensitivity, specificity and accuracy of the urine steroid profile were determined in order to assess information content of such method for the adrenocortical carcinoma diagnosis.

RESULTS: The possibilities of the urine steroid profile determining for the adrenocortical cancer diagnosis are estimated. The method sensitivity was $46.2 \%$, specificity and accuracy were $70.6 \%$ and $60 \%$ respectively. The most reliable of adrenocortical cancer markers were tetrahydro-11-deoxycortisol and dehydroepiandrosterone (38.5\% of cases) increasing concentrations.

CONCLUSIONS: The present study demonstrates relatively low diagnostic efficacy of the urine steroid profile as a primary diagnostic method for adrenocortical cancer determining. This is especially evident in comparison with X-ray diagnostic methods. The technique interpretation is complex and accessible only to specialists with extremely high qualifications. Such fact complicates the distribution and widespread use in clinical practice of this testing method. At the same time the urine steroid profile determination in the future (after additional study) may be apply as an auxiliary diagnostic method which in some cases determines the treatment tactics for patients undergoing adrenocortical cancer adrenalectomy treatment. KEYWORDS: dPheochromocytoma; intraoperative hemodynamic instability; laparoscopic adrenalectomy; Endovascular embolization of preoperative; case report.

KEYWORDS: adrenocortical cancer; steroid profile of urine; gas chromatography-mass spectrometry; steroidogenesis.

\section{ОБОСНОВАНИЕ}

Стероидный профиль мочи (СПМ) является одним из показателей физиологической деятельности надпочечников, яичек и яичников человека. В связи с особенностями секреции стероидных гормонов (например, цикличность в различные часы суток, повышение концентрации в стрессовых ситуациях) оптимальным способом определения СПМ является суточный сбор и дальнейшее исследование аликвоты суточной мочи пациента. Нарушения СПМ отмечаются при отклонениях в развитии, половом созревании и патологии надпочечников (первичном гиперальдостеронизме, гиперкортицизме, врожденной гиперплазии коры надпочечников и адренокортикальном раке) [1, 2]. Измерение СПМ возможно производить несколькими методами, среди которых лидирующее положение занимают высокоэффективная жидкостная хроматография (ВЭЖХ LC-MS/MS - Liquid chromatography-mass spectrometry) и газовая хромато-масс-спектрометрия (ГХ-MC — GC-MS Gas chromatography-mass spectrometry). Оба метода обладают своими достоинствами и недостатками, при этом основным преимуществом ГХ-МС является возможность проведения единовременной оценки всего спектра стероидов (до 60 показателей). К недостаткам ГХ-МС можно отнести достаточно сложные процедуры пробоподготовки и проведения исследования, ограничивающие его применение в рутинной диагностической лаборатории. Подобных недостатков лишен метод ВЭЖХ, однако с его применением возможно проведение одновременного исследования достаточно ограниченного числа стероидов.

Новообразования надпочечников являются достаточно распространенной и чрезвычайно разнородной группой заболеваний. Среди них наибольшую угрозу представляет адренокортикальный рак (АКР). Важную роль в определении злокачественной природы новообразований надпочечников играют лучевые методы исследования. КТ и МРТ позволяют проводить предоперационную диагностику АКР, устанавливать стадию и распространенность злокачественного процесса. Чувствительность и специфичность этих методов оценивается авторами в 93-99,8\% [3-6] и 83,1-98,6\% соответственно [7-9]. Вместе с тем, при высокой диагностической эффективности современных лучевых методов диагностики опухолей надпочечников, для постановки точного клинического диагноза также необходимо проведение гормональных исследований.

Исследование СПМ у пациентов с диагностированными новообразованиями надпочечников предназначено в первую очередь для подтверждения или опровержения АКР. Наличие риска злокачественного поражения надпочечника является ключевым моментом в выборе дальнейшей тактики лечения. В случае подтверждения АКР пациенту показано безотлагательное оперативное лечение. Также исследование СПМ может быть значимо для диагностики отдаленных метастазов АКР после хирургического удаления первичной опухоли надпочечника. При этом в вопросе точности и значимости исследований СПМ в медицинском сообществе к настоящему времени накопилось достаточное количество разногласий. С одной стороны, имеется ряд работ, доказывающих строгую ассоциацию изменений СПМ у пациентов с АКР по нескольким паттернам стероидогенеза [1, 10-12]. В противоположность этому, существует мнение о не столь значимой роли изменения СПМ для диагностики новообразований надпочечников [13-15]. Основными доводами сторонников данной точки зрения являются чрезвычайная морфологическая и функциональная гетерогенность новообразований надпочечни- 
ков, повышенные требования к эксперту в плане трактовки изменений концентрации метаболитов, а также отсутствие достоверности в ее определении.

\section{ЦЕЛЬ}

Настоящее исследование направлено на определение границ точности определения СПМ для диагностики АКР.

\section{МЕТОДЫ}

Дизайн исследования

Характеристика исследования:

- одноцентровое;

- проспективное;

- выборочное;

- неконтролируемое.

\section{Критерии соответствия}

В исследование включены все пациенты независимо от пола и возраста, у которых по данным обследования (КТ брюшной полости) выявлены новообразования надпочечников. Не включались в исследование пациенты с феохромоцитомами, метастатическим поражением надпочечников, мезенхимальными опухолями и кистами надпочечников.

\section{Условия проведения}

Все пациенты проходили лечение в отделении эндокринной хирургии Клиники высоких медицинских технологий им. Н.И. Пирогова Санкт-Петербургского государственного университета (КВМТ СПбГУ).

\section{Продолжительность исследования}

Исследование происходило в период с 2016 по 2018 гг. без смещения запланированных временных интервалов.

\section{Описание медицинского вмешательства}

Всего исследовались 58 пациентов с новообразованиями надпочечников. Для дальнейшего анализа из 58 пациентов были отобраны 30 с адренокортикальными аденомами $(n=17)$ и AKP $(n=13)$, у которых был изучен СПМ. В дальнейшем сопоставление результатов определения СПМ методом ГХ-МС и морфологического исследования образцов новообразований проводилось независимо. 28 больным была выполнена адреналэктомия, двум пациентам проведена биопсия (хирургическое лечение не проводилось в связи с распространенностью опухолевого процесса). Всем пациентам была произведена гистологическая верификация диагноза с оценкой злокачественного потенциала по критериям Weiss (2001) и применением иммуногистохимического (ИГХ) исследования. Диагноз АКР устанавливался при наличии более 5 баллов при световой микроскопии и ИГХ-признаков злокачественного роста. Прогноз рецидива опухоли оценивался по ИГХ (Кі 67).

Методика определения СПМ производилась по нижеприведенной схеме. Проводился забор суточной мочи пациентов. Из суточного объема отбиралась аликвота в 100 мл и замораживалась при $-80{ }^{\circ} \mathrm{C}$. В дальнейшем проводился забор 2 мл мочи через подготовленные прогонкой 4 мл метанола и 2 мл воды картриджи SepPak
(Waters, USA) со скоростью 0,15 мл/мин. Дальнейшие стадии пробоподготовки включали добавление стандартов, гидролиз, водную экстракцию и дериватизацию стероидов с применением триметилсилил имидазола. Следующие этапы - экстракция, вторичная дериватизация и определение уровня стероидов проводились, как описано в методике Н. Тейлора [3]. Качественное и количественное изменение стероидного профиля с определением паттернов, характерных для АКР, оценивалось экспертом (профессор Норман Тейлор, Лондон, Медицинский факультет Королевского Колледжа).

В целях оценки информативности стероидного профиля проводилось определение чувствительности, специфичности и точности методики для диагностики адренокортикальной карциномы. Предшественники адреналового стероидогенеза, изученные в анализируемой группе пациентов, представлены в таблице 1.

Таблица 1. Показатели стероидного профиля мочи, оцениваемые у пациентов с новообразованиями надпочечников

\begin{tabular}{|c|c|c|}
\hline & $\begin{array}{c}\text { Аббревиатура } \\
\text { стероидов }\end{array}$ & Полное название \\
\hline 1 & Androsterone & Андростерон \\
\hline 2 & Aetiocholanolone & Этиохоланон \\
\hline 3 & $\mathrm{DHA}$ & Дегидроэпиандростерон \\
\hline 4 & $110 x o A e$ & 11-оксиэтиохоланон \\
\hline 5 & $11 \mathrm{~A}$ & 11-гидроксиандростерон \\
\hline 6 & $11 \mathrm{Ae}$ & 11-гидроксиэтиохоланон \\
\hline 7 & 16a DHA & 16а-гидроксидегидроэпиандростерон \\
\hline 8 & $\mathrm{P} 2$ & Прегнандиол \\
\hline 9 & P3 & Прегнантриол \\
\hline 10 & DA3 & Андростентриол \\
\hline 11 & THE & Тетрагидрокортизон \\
\hline 12 & THA & Тетрагидро-11-дегидрокортикостерон \\
\hline 13 & THB & Тетрагидрокортистерон \\
\hline 14 & alloTHB & Алло-тетрагидрокортистерон \\
\hline 15 & THF & Тетрагидрокортизол \\
\hline 16 & alloTHF & Алло-тетрагидрокортизол \\
\hline 17 & a Cortolone & а-Кортолон \\
\hline 18 & b Cortol & b-Кортол \\
\hline 19 & b Cortolone & b-Кортолон \\
\hline 20 & a Cortol & а-Кортол \\
\hline 21 & $17 a-d A 2$ & Андростендиол-17а \\
\hline 22 & $17 b-d A 2$ & Андростендиол-17b \\
\hline 23 & $\mathrm{dP} 2$ & Прегнендиол \\
\hline 24 & $\mathrm{dP3}$ & Прегнентриол \\
\hline 25 & $110 x \circ \mathrm{P} 3$ & 11-оксопрегнантриол \\
\hline 26 & $17 \mathrm{OHP}$ & 17-гидроксипрегненолон \\
\hline 28 & 16 Epi A & 16а-гидроксиэпиандростерон \\
\hline 29 & THS & Тетрагидро-11-дезоксикортизол \\
\hline 30 & $\mathrm{dP} 3 a, 16 a, 20 a$ & 5-прегнен-3a,16a,20-триол \\
\hline 31 & $16 \mathrm{adP}$ & 16а-гидроксипрегненолон \\
\hline 32 & $d P 3 \beta, 16 a, 20 \beta$ & 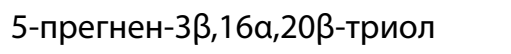 \\
\hline 33 & $21 \mathrm{dP}$ & 21-гидроксипрегненолон \\
\hline 34 & $\mathrm{dP} 3 \beta, 20 a, 21$ & 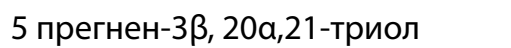 \\
\hline
\end{tabular}




\section{Основной исход исследования}

Оценивались показатели диагностической эффективности СПМ для диагностики АКР: точность, специфичность и чувствительность.

\section{Анализ в подгруппах}

Пациенты были разделены на 2 подгруппы в зависимости от наличия у них злокачественного новообразования надпочечника: адренокортикальных аденом $(\mathrm{n}=17)$ и $\operatorname{AKP}(n=13)$.

\section{Методы регистрации исходов}

Для оценки стероидного профиля мочи использовался масс-спектрометр Shimadzu 2060. Хирургическое лечение пациентов проводилось с использованием эндовидеохирургической техники Karl Storz (Германия).

\section{Этическая экспертиза}

Протокол Комитета по биомедицинской этике Клиники высоких медицинских технологий им. Н.И. Пирогова (N 12/15, подписан 14.12.15 г.). При проведении этической экспертизы по представленным документам замечаний нет. Одобрено проведение исследования на базе Клиники высоких медицинских технологий им. Н.И. Пирогова.

\section{Статистический анализ}

Размер выборки предварительно не рассчитывался.

Методы статистического анализа данных. При анализе данных использована статистическая программа Microsoft Excel (компания Microsoft, США). Концентрации стероидов фиксировались в электронной базе данных. В дальнейшем оценивалась диагностическая эффективность методики определения СПМ для диагностики АКР: точность, чувствительность, эффективHOCTь.

\section{РЕЗУЛЬТАТЫ}

Объекты (участники) исследования

Всего исследовались 62 пробы у 58 пациентов с новообразованиями надпочечников. Исследование носило характер слепого проспективного. Для дальнейшего анализа из 58 пациентов были отобраны 30 с адренокортикальными аденомами $(n=17)$ и AKP $(n=13)$, у которых был изучен СПМ. Необходимо отметить, что при изучении стероидного профиля диагноз известен не был, и сопоставление результатов определения СПМ методом ГХ-МС и морфологического исследования образцов новообразований проводилось независимо. Распределение по полу: 10 мужчин и 20 женщин. Средний возраст составил 46,8土12,7 года (24-70 лет). 28 больным была выполнена адреналэктомия, двум пациентам проведена биопсия (хирургическое лечение не проводилось в связи с распространенностью опухолевого процесса). Клинические и лабораторно инструментальные данные фиксировались в истории болезни и в дальнейшем заносились в электронную базу данных. Всем пациентам была произведена гистологическая верификация диагноза с оценкой злокачественного потенциала по критериям Weiss (2001) и применением ИГX-исследования.

\section{Основные результаты исследования}

Критериями злокачественного преобразования опухоли надпочечника служили нарушения паттерна масс-спектров комплекса стероидов, выявляемых методом ГХ-МС (рис. 1, 2). Основным показателем злокачественного преобразования опухоли является увеличение концентрации тетрагидро-11-дезоксикортизола (THS), дегидроэпиандростерона, прегнен-3ß,16a,20a-триола, прегнен-3a,16a,20a-триола, прегнандиола, прегнантриола и прегнентриола в моче (табл. 2).

Таблица 2. Концентрация исследованных стероидов, показавших наибольшие отличия в различных группах пациентов, мкг/л

\begin{tabular}{|c|c|c|}
\hline \multirow[b]{2}{*}{ Стероиды } & \multicolumn{2}{|c|}{ Нозологии } \\
\hline & $\begin{array}{c}\text { Адренокортикальный рак } \\
\qquad(n=13)\end{array}$ & $\begin{array}{c}\text { Аденома надпочечника } \\
(\mathbf{n = 1 7 )}\end{array}$ \\
\hline Тетрагидро-11-дезоксикортизол (THS) & $2304 \pm 802$ & $14 \pm 361$ \\
\hline Дегидроэпиандростерон (DHA) & $4382 \pm 7786$ & $46 \pm 69$ \\
\hline 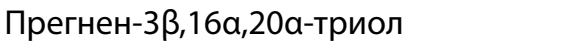 & $209 \pm 324$ & $5 \pm 2,4$ \\
\hline Прегнен-3a,16a,20a-триол & $311 \pm 383$ & $62 \pm 34$ \\
\hline Прегнандиол (Р2) & $552 \pm 1702$ & $181 \pm 129$ \\
\hline Прегнантриол (Р3) & $625 \pm 1152$ & $314 \pm 152$ \\
\hline Прегнентриол ( $\Delta$ Р3) & $1228 \pm 2887$ & $80 \pm 51$ \\
\hline
\end{tabular}




\section{A Ivanov 12}

2/random, all + 2 MOTSIM

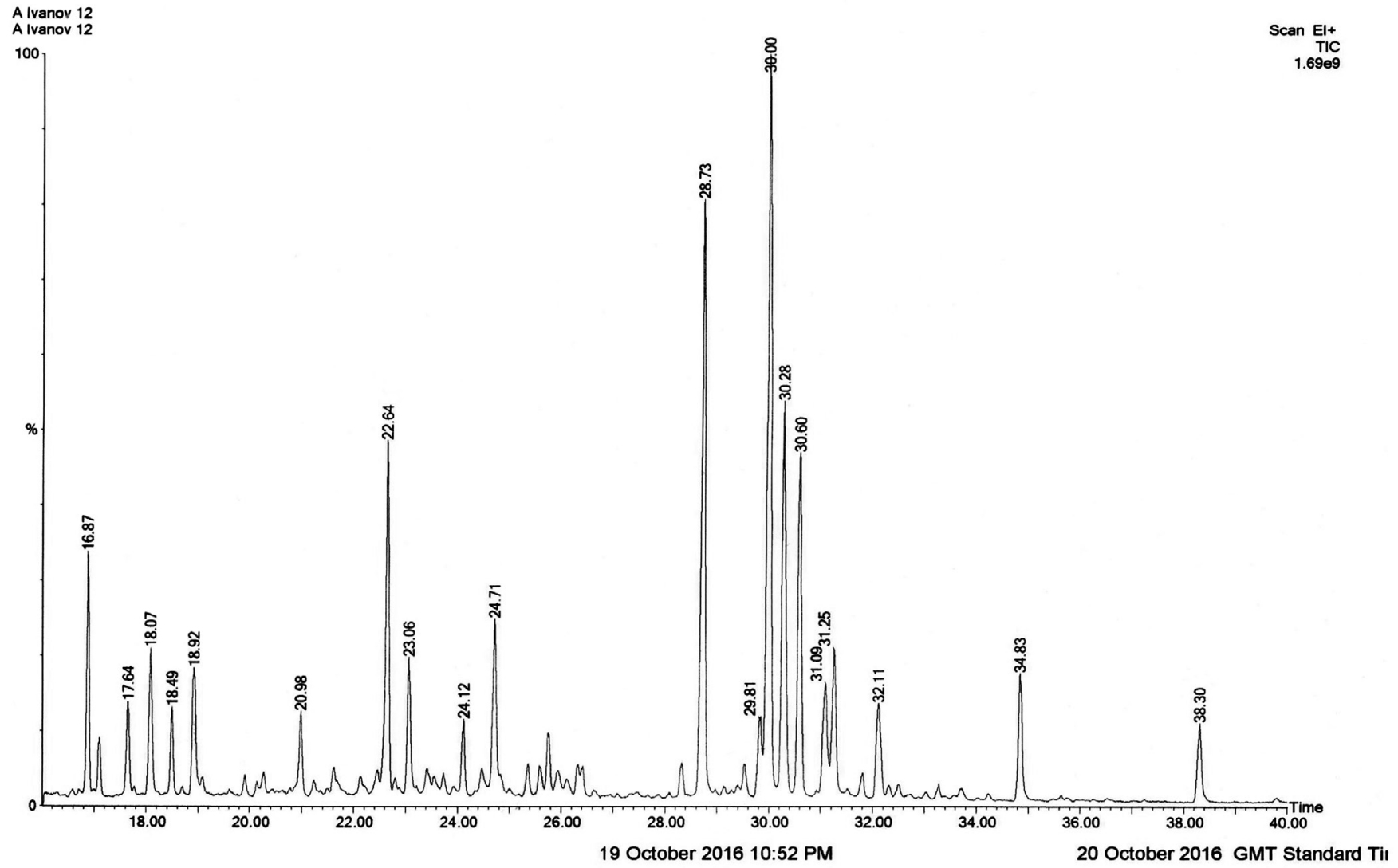

Рисунок 1. Пример стероидного профиля суточной мочи больного аденомой надпочечника (20.98 - 11 oxoAe; 22.64 - 11A; 24.12 - P2; 24.71 - P3; 28.73 - THE; 30.00 - THF).

\section{A1 06-18 6}

\section{$2 / \mathrm{r} 1 / 2+2$ MOTSIM}

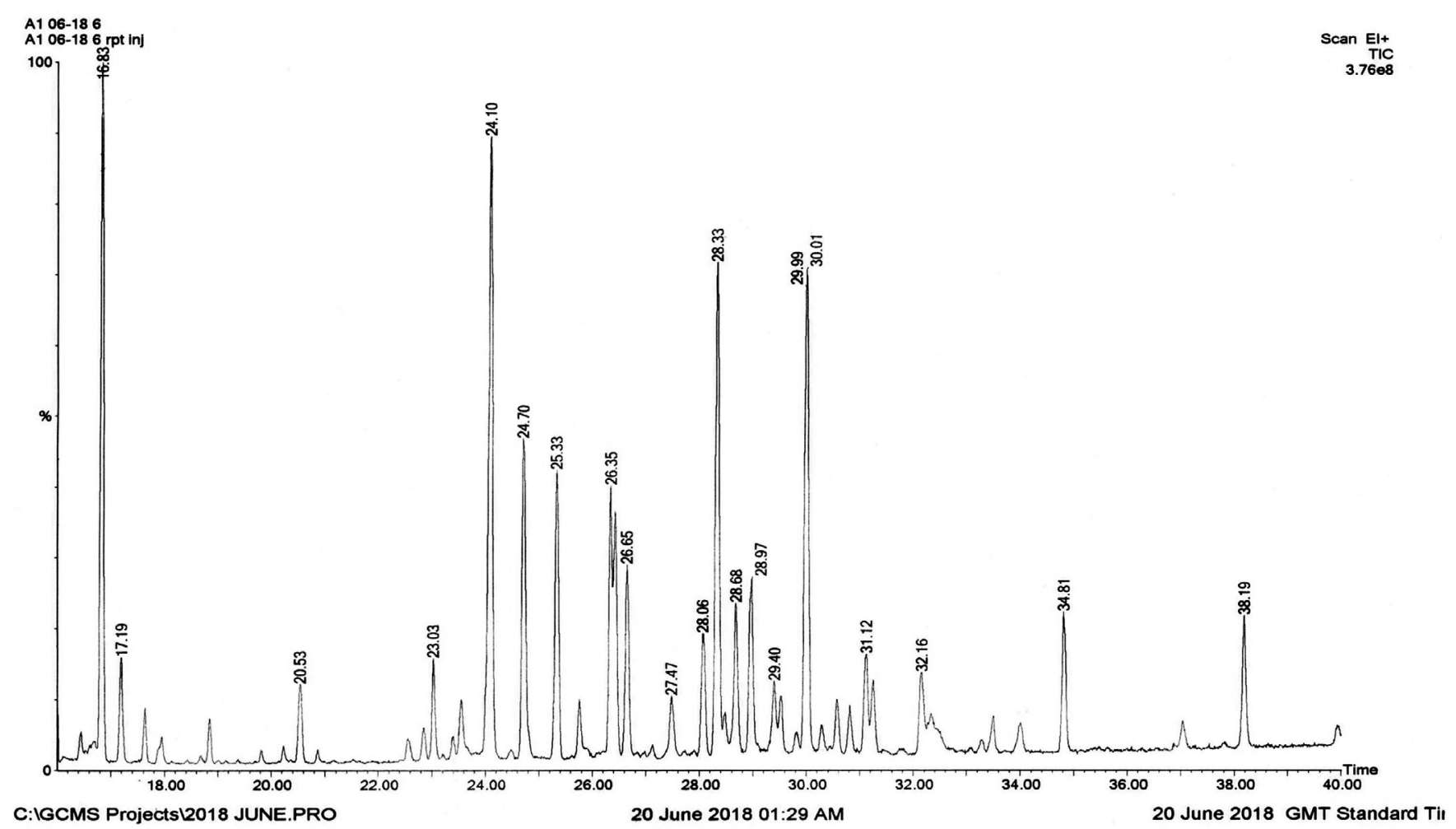

Рисунок 2. Пример стероидного профиля суточной мочи больного адренокортикальным раком (AKP: 24.10 - P2; 24.70 - P3; 25.33 - dP2; 26,35 - THS; 28.33 - dP3; 30.01 - THF). 
Как следует из нашего исследования (табл. 3), наиболее достоверными маркерами АКР становится повышение концентрации THS (2 случая) и дегидроэпиандростерона (2 случая) или их сочетание (1 наблюдение). Таким образом, нарушения их соотношения с другими стероидами отмечаются в 5 случаях из 13 проанализированных (38,5\%).

Одним из ключевых диагностических критериев при анализе СПМ является соотношение концентраций прегнандиола (P2) и прегнантриола (Р3). Подобная картина является хорошим маркером АКР у мужчин и женщин в постменопаузе. У женщин репродуктивного возраста увеличение концентрации Р2 и Р3 может быть обусловлено фазой менструального цикла.
Наиболее востребованной для настоящего исследования была оценка диагностической эффективности изучения СПМ для диагностики АКР. В результате из 13 случаев гистологически подтвержденных АКР только 6 (46,2\%) продемонстрировали паттерн СПМ с характерными изменениями. В оставшихся 7 случаях (53,8\%) достоверных биохимических признаков злокачественного перерождения тканей надпочечников не было выявлено (табл. 3, 4).

Интересен анализ СПМ, когда новообразования надпочечников оказывались доброкачественными опухолями. Из 17 случаев в 12 (70,6\%) продемонстрировано четкое отсутствие маркеров АКР, а в пяти наблюдениях (29,4\%) были выявлены сомнения, которые не позволили достоверно исключить злокачественную

Таблица 3. Изменения в стероидном профиле мочи, выявленные экспертом у больных АКР

\begin{tabular}{|c|c|c|c|}
\hline 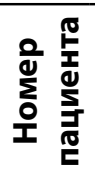 & $\begin{array}{c}\text { Изменения стероидного } \\
\text { профиля у пациентов с АКР }\end{array}$ & $\begin{array}{c}\text { Заключение эксперта } \\
\text { по данным ГХ-МС }\end{array}$ & $\begin{array}{c}\text { Сравнение с результатами } \\
\text { морфологических исследований } \\
\text { (верно/неверно) }\end{array}$ \\
\hline 1 & $\begin{array}{l}\text { Нарушено соотношение Р2 и Р3, } \\
\text { 3a16a20а и } \triangle \text { P3 повышены }\end{array}$ & Рака нет & Неверно \\
\hline 2 & $\triangle \mathrm{A} 3$ повышен & Рака нет & Неверно \\
\hline 3 & Нарушено соотношение Р2 и Р3 & $\begin{array}{l}\text { Некоторые подозрения, } \\
\text { но четкого ответа не дано }\end{array}$ & Неверно \\
\hline 4 & $\begin{array}{l}\Delta \text { Р3 повышен, повышено } \\
\text { соотношение 3ß20а21 }\end{array}$ & $\begin{array}{l}\text { Некоторые подозрения, } \\
\text { но четкого ответа не дано }\end{array}$ & Неверно \\
\hline 5 & $\begin{array}{l}\text { DHA повышен, отношение } 20 \beta / 20 a \\
\text { прегнен-3ß16a20 повышено }\end{array}$ & Рак есть & Верно \\
\hline 6 & P2, P3, THS, $\triangle$ P3 повышены & Рак есть & Верно \\
\hline 7 & Маркеров рака не выявлено & $\begin{array}{l}\text { Некоторые подозрения, } \\
\text { но четкого ответа не дано }\end{array}$ & Неверно \\
\hline 8 & THS, DHA, $\triangle$ P3 повышены & Рак есть & Верно \\
\hline 9 & Маркеров рака не выявлено & $\begin{array}{c}\text { Некоторые подозрения, } \\
\text { но четкого ответа не дано }\end{array}$ & Неверно \\
\hline 10 & $\begin{array}{l}\text { 5a/5 } \beta \text { Androsterone нарушено } \\
\text { соотношение, THS повышен }\end{array}$ & Рак есть & Верно \\
\hline 11 & DHA повышен, $\triangle$ P3 повышен, THS N & Рак есть & Верно \\
\hline 12 & Маркеров рака не выявлено & Рака нет & Неверно \\
\hline 13 & P2, P3, THS, $\triangle$ P3 повышены & Рак есть & Верно \\
\hline
\end{tabular}

Таблица 4. Результаты изучения стероидного профиля мочи в группе пациентов с адренокортикальным раком ( $\mathrm{n}=13)$ для определения канцерогенеза

\section{Истинноположительные результаты (\%)}

\author{
Ложноотрицательные \\ результаты (\%)
}
$6(46,2 \%)$
$7(53,8 \%)$ 
опухоль (табл. 5). Такие результаты были расценены как ложноположительные (табл. 6).

В целом в изучаемой группе больных $(n=30)$ чувствительность и специфичность в диагностике АКР составляет $46,2 \%$ и 70,6\% соответственно. Точность методики была определена, как $60 \%$.

\section{Дополнительные результаты исследования} Дополнительных результатов не отмечено. Нежелательные явления не отмечены.

\section{ОБСУЖДЕНИЕ}

Резюме основного результата исследования

В целом исследование демонстрирует значительно меньшую ассоциацию стероидных маркеров АКР в СПМ с гистологически подтвержденным диагнозом в каждом конкретном случае. Несмотря на то что некоторые из стероидных маркеров АКР подтверждают часть случаев, общая картина не позволяет поставить точный диагноз, опираясь только на данные ГХ-МС.

Таблица 5. Изменения в стероидном профиле мочи, выявленные экспертом у пациентов с аденомой надпочечника

\begin{tabular}{|c|c|c|c|}
\hline 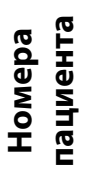 & $\begin{array}{c}\text { Изменения стероидного } \\
\text { профиля у больных аденомой } \\
\text { надпочечника }\end{array}$ & $\begin{array}{c}\text { Заключение эксперта по } \\
\text { данным } \\
\text { ГХ-МС }\end{array}$ & $\begin{array}{c}\text { Сравнение с результатами } \\
\text { морфологических } \\
\text { исследований } \\
\text { (верно/неверно) }\end{array}$ \\
\hline 1 & $\mathrm{~N}$ & Рака нет & Верно \\
\hline 2 & $\mathrm{~N}$ & Рака нет & Верно \\
\hline 3 & $\mathrm{~N}$ & Рака нет & Верно \\
\hline 4 & $\begin{array}{l}\text { N, есть изменения в синтезе } \\
\text { половых гормонов }\end{array}$ & Точного ответа не дано & Неверно \\
\hline 5 & $\mathrm{~N}$ & Рака нет & Верно \\
\hline 6 & $\mathrm{~N}$ & Рака нет & Верно \\
\hline 7 & $\mathrm{~N}$ & Рака нет & Верно \\
\hline 8 & P2/P3 нарушено соотношение & $\begin{array}{c}\text { Некоторые подозрения, но четкого } \\
\text { ответа не дано }\end{array}$ & Неверно \\
\hline 9 & $\mathrm{~N}$ & $\begin{array}{c}\text { Некоторые подозрения, но четкого } \\
\text { ответа не дано }\end{array}$ & Неверно \\
\hline 10 & $\mathrm{~N}$ & Рака нет & Верно \\
\hline 11 & $\begin{array}{l}\text { N, есть изменения в синтезе } \\
\text { половых гормонов }\end{array}$ & Рака нет & Верно \\
\hline 12 & $\mathrm{~N}$ & Рака нет & Верно \\
\hline 13 & $\begin{array}{l}\text { P2, P3, } \triangle \text { P3 незначительно } \\
\text { повышены, иных маркеров рака нет }\end{array}$ & $\begin{array}{c}\text { Некоторые подозрения, но четкого } \\
\text { ответа не дано }\end{array}$ & Неверно \\
\hline 14 & THS повышен & Рак есть & Неверно \\
\hline 15 & $\mathrm{~N}$ & Рака нет & Верно \\
\hline 16 & $\mathrm{~N}$ & Рака нет & Верно \\
\hline 17 & Маркеров рака нет & Рака нет & Верно \\
\hline
\end{tabular}

Таблица 6. Результаты изучения стероидного профиля мочи в группе пациентов с аденомами надпочечника ( $\mathrm{n}=17$ ) для определения прогноза канцерогенеза

\section{Истинноотрицательные результаты (\%)}

\section{Ложноположительные результаты (\%)}
$12(70,6 \%)$
$5(29,4 \%)$ 
Обсуждение основного результата исследования

Подобные критерии также описаны ранее в исследованиях Taylor N., 2013 и Velikanova et al., 2016 [16-18]. Основным показателем является увеличение концентрации THS в моче.

По данным ряда исследований было выявлено повышение уровня THS при развитии злокачественных новообразований коркового слоя надпочечников $[19,20]$. Данное вещество является продуктом метаболизма 11-дезоксикортизола, прямого предшественника кортизола. Увеличение концентрации THS в моче при канцерогенезе может быть связано с нарушением функции 11ß-гидроксилазы. Это митохондриальный фермент из суперсемейства цитохрома P450, преимущественно локализованный в митохондриях клеток пучковой зоны коры надпочечников. Его функцией является добавление гидроксильной группы к 11-деоксикортизолу и 11-деоксикортикостеронувпозиции 11-гоатомауглерода, в результате чего образуются кортизол и кортикостерон соответственно. При прекращении или нарушении функционирования данного гена происходит накопление 11-дезоксикортизола, который затем метаболизируется в THS и его стереоизомер 5a-allo-THS и выводится с мочой. При сохранении функций 11ß-гидроксилазы происходит свободный синтез кортизола и его дальнейший метаболизм до кортизона при участии гидроксистероиддегидрогеназы $11 \beta$. В дальнейшем эти оба стероида метаболизируются до тетрагидро-восстановленных производных: тетрагидрокортизола (THF) и тетрагидрокортизона (THE) и их 5а-стереоизомеров (allo-THF и allo-THE соответственно). Метаболизм снижает биологическую активность гормонов и увеличивает их растворимость в воде путем преобразования их в гидрофильные соединения, которые могут выводиться с мочой. Именно THF и THE преобладают в нормальном стероидном профиле.

В целом в изучаемой группе больных $(n=30)$ чувствительность и специфичность в диагностике АКР составляют $46,2 \%$ и 70,6\% соответственно. Точность методики была определена как $60 \%$.

В результате исследования произведено уточнение критериев злокачественности при изучении СПМ методом ГХ-МС. До настоящего момента наиболее точным критерием злокачественности новообразования надпочечников считалось увеличение экскреции с мочой THS. B работе Kerkhofs et al. (2015) демонстрируется 100\% чувствительность данного показателя при 99\% специфичности [10]. Более осторожная оценка приводится Velikanova et al. (2016) [18]. Специфичность увеличения концентрации в моче только THS, без учета дополнительных параметров, составляет 74,2\%. На первое место по значимости этими авторами выводятся сочетание увеличения концентраций THS или DHA, а также его метаболитов 17-dA2, 16-OH- DHA и dA3, и значительного смещения соотношения $3 a, 16,20 \mathrm{dP} 3 / 3 \beta, 16,20 \mathrm{dP} 3$ в сторону $3 \beta, 16,20 d P 3$. Поэтому при анализе СПМ различными методами необходимо проводить детектирование и сравнительную оценку концентрации различных 5-ен-прегненов: 16-ОН-прегненолон (16dP), 21-ОН-прегненолон (21dP), 21-ОН-прегнендиол (21dP2), 11-гидроксипрегнентриол. Помимо вышеперечисленных, в качестве маркеров АКР исследователями отмечается достаточно обширный список стероидов, таких как этиохоланолон, 16-оксо-андростендиол, прегнандиол, прегнантриол (P3), 11-оксо-Р3, 6ß-ОН-прегнанолон, прегнендиол (dP2), 3a,17,20-прегнентриол (3adP3), 3ß,17,20-прегнентриол (3ßdP3), 5-ен-прегненов 16dP и $21 \mathrm{dP}[10,20]$. Авторами было отмечено, что рассматривать изменения паттерна стероидного профиля следует только в комплексе, выявляя совместные колебания уровней различных компонентов. Подобный подход не позволяет исключить субъективность описываемой методики, связанную с условиями проведения анализа и интерпретацией результата.

В качестве важной особенности стероидогенеза следует отметить выявленное во многих случаях повышение уровня Р2 и Р3 у женщин репродуктивного возраста. Несмотря на то что данные показатели являются маркерами АКР для мужчин и женщин в постменопаузе, их наличие в суточной моче может быть обусловлено характером синтеза в половых железах в зависимости от фазы менструального цикла. Это также зачастую затрудняет интерпретацию спектрограмм (см. табл. 5), особенно в случаях с доброкачественными аденомами надпочечника (отмечено в 2 из 17 наблюдений - 11,8\%).

\section{Ограничения исследования}

Ограничением метода является его субъективность, связанная с тем, что интерпретация методики сложна и доступна лишь специалистам с чрезвычайно высокой квалификацией.

\section{ЗАКЛЮЧЕНИЕ}

Настоящее исследование показало низкую чувствительность (46,2\%) и невысокую точность (60\%) определения СПМ как метода первичной диагностики АКР. Особенно это видно по сравнению с лучевыми методами диагностики. Интерпретация методики сложна и доступна лишь специалистам с чрезвычайно высокой квалификацией, что затрудняет распространение и широкое применение этого метода обследования в клинической практике. Вместе с тем определение СПМ в перспективе (после дополнительного изучения) может быть использовано как вспомогательный метод диагностики, в ряде случаев определяющий лечебную тактику у пациентов, перенесших адреналэктомию по поводу АКР.

\section{ДОПОЛНИТЕЛЬНАЯ ИНФОРМАЦИЯ}

Источник финансирования. Исследование выполнено при финансовом обеспечении Санкт-Петербургского государственного университета.

Конфликт интересов. Авторы декларируют отсутствие конфликта интересов.

Участие авторов. Концепция и дизайн исследования - Иванов А.В., Краснов Л.М., Русаков В.Ф., Черников Р.А., Федотов Ю.Н., Щербаков И.Е., Саблин И.В., Слепцов И.В., Реброва Д.В., Уварова М.А.; сбор и обработка материалов - Саблин И.В., Федоров Е.А., Реброва Д.В., Уварова М.А., Иванов А.В.; анализ полученных данных, написание текста Русаков В.Ф., Щербаков И.Е., Реброва Д.В., Уварова М.А., Иванов А.В., Краснов Л.М.

Благодарности. Авторы выражают благодарность: профессору Норману Тейлору, Лондон, Медицинский факультет Королевского Колледжа. 


\section{СПИСОК ЛИТЕРАТУРЫ | REFERENCES}

1. Шафигуллина 3. Р., Великанова Л.И., Ворохобина Н.В. и др. Диагностическое значение стероидного профиля биологических жидкостей у больных с синдромом Иценко-Кушинга // Проблемы эндокринологии. - 2015. - Т.61. - №4. - C. 4-8. [Shafigullina ZR, Velikanova LI, Vorokhobina NV, et al. The diagnostical importance of steroid profiles of biological fluids of patients with Cushing's syndrome. Problems of Endocrinology. 2015;61(4):4-8. (In Russ).] doi: 10.14341/probl20156144-8.

2. Галахова Р.К., Великанова Л.И., Ворохобина Н.В., и др. Информативность различных лабораторных технологий в диагностике заболеваний гипофизарно адреналовой системы у больных с гиперплазией коры надпочечников и артериальном гиперкортицизме // Вестник Санкт-Петербургской медицинской академии последипломного образования. - 2011. - Т.3. №1. - C. 43-49. [Galakhova RK, Velikanov LI, Vorokhobina NV, et al. Descriptiveness of various laboratory technologies in diseases pituitary-adrenal system diagnosis in patients with adrenal hyperplasia and arterial hypertension. Bulletin of St-Petersburg Medical Academy of Postgraduate Studies. 2011;3(1):43-49. (In Russ).]

3. Tauchmanova L, Rossi R, Biondi B, et al. Patients with subclinical Crushing's syndrome due to adrenal adenoma have increased cardiovascular risk. J Clin Endocrinol Metab. 2002;87(11):4872-4878. doi: 10.1210/jc.2001-011766.

4. Руммени Э.Й., Кармазановский Г.К. Магнитно-резонансная томография тела. - М.: Медицинская литература; 2014. [Rummeni El, Karmazanovsky GK. Magnitno-rezonansnaya tomografiya tela. Moscow: Meditsinskaya literatura; 2014. (In Russ).]

5. Степанова Ю.А., Тимина И.Е., Ашивкина О.И., и др. Опухоли надпочечников: клинико-лучевая диагностика // Медицинская визуализация. - 2014. - №2. - C. 48-60. [Stepanova YuA, Timina IE, Ashivkina Ol, et al. Adrenal glands tumours: clinicalradiology diagnostics (literature review). Medical visualization. 2014;(2):48-60. (In Russ).]

6. Садриев О.Н., Гаибов А.Д., Анварова Ш.С. Диагностика и хирургическое лечение синдрома Конна // Вестник Авиценны. 2014. - №2. - C. 47-52. [Sadriev ON, Gaibov AD, Anvarova SS. Diagnosis and surgical treatment of Conn's syndrome. Bulletin of Avicenna. 2014;(1):47-52. (In Russ).]

7. Willatt JM, Francis IR. Radiologic evaluation of incidentally discovered adrenal masses. Am Fam Physician. 2010;81(11):1361-1366.

8. Kapoor A, Morris T, Rebello R. Guidelines for the management of the incidentally discovered adrenal mass. Can Urol Assoc J. 2011;5(4):241-247. doi: 10.5489/cuaj.11135. Erratum in: Can Urol Assoc J. 2012;6(4):244
9. Song JH, Mayo-Smith WW. Current status of imaging for adrenal gland tumors. Surg Oncol Clin N Am. 2014;23(4):847-861. doi: 10.1016/j.soc.2014.06.002.

10. Kerkhofs TM, Kerstens MN, Kema IP, et al. Diagnostic value of urinary steroid profiling in the evaluation of adrenal tumors. Horm Cancer. 2015;6(4):168-175. doi: 10.1007/s12672-015-0224-3.

11. Arlt W, Biehl M, Taylor AE, et al. Urine steroid metabolomics as a biomarker tool for detecting malignancy in adrenal tumors. J Clin Endocrinol Metab. 2011;96(12):3775-3784. doi: 10.1210/jc.2011-1565.

12. Stigliano A, Chiodini I, Giordano R, et al. Managment of adrenocortical carcinoma consensus statementof the Italian Society of Endocrinology (SIE). J Endocrinol Invest. 2016;39(1):103-121. doi: 10.1007/s40618-015-0349-9.

13. Calissendorff J, Calissendorff F, Falhammar H. Adrenocortical cancer: mortality, hormone secretion, proliferation and urine steroids experience from a single centre spanning three decades. BMC Endocr Disord. 2016;16:15. doi: 10.1186/s12902-016-0095-9.

14. Fassnacht $M$, Kroiss M, Allolio B. Update in adrenocortical carcinoma. J Clin Endocrinol Metab. 2013;98(12):4551-4564. doi: 10.1210/jc.2013-3020.

15. Tiu SC, Chan AO, Taylor NF, et al. Use of urinary steroid profiling for diagnosing and monitoring adrenocortical tumours. Hong Kong Med J. 2009;15(6):463-470.

16. Taylor DR, Ghataore L, Couchman L, et al. A 13-steroid serum panel based on LC-MS/MS: use in detection of adrenocortical carcinoma. Clin Chem. 2017;63(12):1836-1846. doi: 10.1373/clinchem.2017.277624.

17. Taylor NF. Urinary steroid profiling. Methods Mol Biol. 2013;1065:259-276. doi: 10.1007/978-1-62703-616-0_17.

18. Velikanova LI, Shafigullina ZR, Lisitsin AA, et al. Different types of urinary steroid profiling obtained by high-performance liquid chromatography and gas chromatography-mass spectrometry in patients with adrenocortical carcinoma. Horm Cancer. 2016;7(5-6):327-335. doi: 10.1007/s12672-016-0267-0.

19. Okihara R, Mitamura K, Hasegawa M, et al. Potential corticoid metabolites: chemical synthesis of 3- and 21-monosulfates and their double-conjugates of tetrahydrocorticosteroids in the 5alphaand 5beta-series. Chem Pharm Bull (Tokyo). 2010;58(3):344-353. doi: $10.1248 / c p b .58 .344$.

20. Minowada S, Kinoshita K, Hara M, et al. Measurement of urinary steroid profile in patients with adrenal tumor as a screening method for carcinoma. Endocrinol Jpn. 1985;32(1):29-37. doi: 10.1507/endocrj1954.32.29.

\section{ИНФОРМАЦИЯ ОБ АВТОРАХ [AUTHORS INFO]}

*Щербаков Илья Евгеньевич, к.м.Н., хирург [llya E. Shcherbakov, MD, PhD], Санкт-Петербургский государственный университет, Клиника высоких медицинских технологий им. Н.И. Пирогова, Санкт-Петербург, Россия, ул. Фонтанка, д. 154 [address, Fontanka emb, 154, St. Petersburg, Russia]; телефон: 8 (960) 230-89-36; ORCID: https://orcid.org/ 0000-0001-8311-0464; eLibrary SPIN: e-mail: ies77post@gmail.com

Черников Роман Анатольевич, д.м.н., заведующий хирургическим отделением, Санкт-Петербургский государственный университет, Клиника высоких медицинских технологий им. Н.И. Пирогова [Roman A. Chernikov, MD, PhD]; телефон: +79219800099; ORCID: https://orcid.org/0000-0002-3001-664X; eLibrary SPIN: 7093-1088; e-mail: yaddd@yandex.ru

Русаков Владимир Федорович, к.м.н., врач-эндокринолог, Санкт-Петербургский государственный университет, Клиника высоких медицинских технологий им. Н.И. Пирогова [Vladimir F. Rusakov, MD, PhD]; телефон: 8 (921) 375-48-90; eLibrary SPIN: 1345-3530; e-mail: rusvf@mail.ru

Федоров Елисей Александрович, к.м.н., хирург, Санкт-Петербургский государственный университет, Клиника высоких медицинских технологий им. Н.И. Пирогова [Elysey A. Fedorov, MD, PhD]; телефон: 8 (911) 299-71-43; eLibrary SPIN: 5673-2633; e-mail: elick@mail.ru

Федотов Юрий Николаевич, д.м.н., директор Клиники высоких медицинских технологий им. Н.И. Пирогова [Uriy N. Fedotov, MD, PhD]; телефон: 8 (812) 676-25-07; eLibrary SPIN: 4833-3371; e-mail: info@gosmed.ru

Саблин Илья Владимирович, хирург, Санкт-Петербургский государственный университет, Клиника высоких медицинских технологий им. Н.И. Пирогова [Ilya V. Sablin, MD]; телефон: 8 (981) 708-76-16;

ORCID: https://orcid.org/0000-0001-7912-4580; eLibrary SPIN: 5479-0942 ; e-mail: sablin_ilya@mail.ru 
Чинчук Игорь Константинович, к.м.н., хирург, Санкт-Петербургский государственный университет, Клиника высоких медицинских технологий им. Н.И. Пирогова [Igor K. Chinchuk, MD, PhD]; телефон: 8 (921) 383-83-14; eLibrary SPIN: 6252-6710; e-mail: Zuldjin@mail.ru

Слепцов Илья Валерьевич, д.м.н., хирург, Санкт-Петербургский государственный университет, Клиника высоких медицинских технологий им. Н.И. Пирогова [Ilya V. Sleptcov, MD, PhD]; телефон: +7 (921) 9517088 ;

ORCID: https://orcid.org/0000-0002-1903-5081; eLibrary SPIN: 2481-4331; e-mail: newsurgery@yandex.ru

Краснов Леонид Михайлович, д.м.н., начальник научно-образовательного отдела, Санкт-Петербургский государственный университет, Клиника высоких медицинских технологий им. Н.И. Пирогова [Leonid M. Krasnov, MD, PhD]; телефон: 8 (921) 31429 79; ORCID: 0000-0003-4449-0251; eLibrary SPIN: 355848; e-mail: krasnov.surg@mail.ru

Реброва Дина Владимировна, к.м.н., врач-эндокринолог, Санкт-Петербургский государственный университет, Клиника высоких медицинских технологий им. Н.И. Пирогова [Dina V. Rebrova MD, PhD]; телефон: 8 (911) 239 22 $09 ;$ ORCID: 7840-4174; eLibrary SPIN: 6284-9008; e-mail:

Уварова Марина Александровна, биолог, отделение гематологии, Санкт-Петербургский государственный университет, Клиника высоких медицинских технологий им. Н.И. Пирогова [Marina A. Uvarova] ORCID: https://orcid.org/0000-0002-4000-0836; e-mail: Angie_@inbo.ru

Иванов Андрей Владимирович, к.б.н., заведующий отделом генетики, Санкт-Петербургский государственный университет, Клиника высоких медицинских технологий им. Н.И. Пирогова [Andrey V. Ivanov, PhD] телефон: 8 (921) 971-97 37; ORCID: https://orcid.org/0000-0002-16767754; eLibrary SPIN: 8271-3596; e-mail: anivanov@omrb.pnpi.spb.ru

\section{ИНФОРМАЦИЯ:}

Рукопись получена: 11.02.2020. Одобрена к публикации: 13.02.2020. Опубликована online: 11.02.2020 Received: 04.03.2020 . Accepted: 13.02.2020 . Published online: 11.02.2020.

\section{ЦИТИРОВАТЬ:}

Щербаков И.Е., Черников Р.А., Русаков В.Ф., Федоров Е.А., Федотов Ю.Н., Саблин И.В., Чинчук И.К., Слепцов И.В., Краснов Л.М., Реброва Д.В., Уварова М.А., Иванов А.В. Современные возможности применения стероидного профиля мочи для диагностики адренокортикального рака // Эндокринная хирургия — 2020. — Т. 14. — №1. — С. 4-13. doi: https://doi.org/10.14341/serg12307

\section{TO CITE THIS ARTICLE:}

Shcherbakov IE, Chernikov RA, Rusakov VF, Fedorov EA, Fedotov YN, Sablin IV, Chinchuk IK, Sleptcov IV, Krasnov LM, Rebrova DV, Uvarova MA, Ivanov AV. Modern possibilities of the urine steroid profile testing applying for the adrenocortical cancer diagnosis. Endocrine surgery. 2020;14(1): 4-13. doi: https://doi.org/10.14341/serg12307 